\title{
EIT Image Reconstruction Using Iterative TV Regularized PD-IPM Algorithm
}

\author{
Md. Rabiul Islam ${ }^{*}$ and Md. Adnan Kiber ${ }^{2}$ \\ ${ }^{1,2}$ Department of Electrical and Electronics Engineering \\ University of Dhaka \\ rabiul.du18@gmail.com
}

\begin{abstract}
Biological tissues have electrical conductivity and permittivity properties which depend on tissue composition, structure and health status. Bioelectrical properties can be utilized for non invasive diagnosis. Electrical Impedance Tomography (EIT) is a method for reconstructing the image of distribution of electrical conductivity and permittivity inside a volume from measurements made at the surface of the volume. EIT image reconstruction is an ill-posed problem that requires a priori information called regularization. The Total Variation (TV) regularization is often used in solving EIT inverse problem. In this paper, simulation has been carried out in noise free and noisy cases and TV regularized iterative Primal Dual Interior Point Method (PD-IPM) has been used to reconstruct the difference conductivity image.
\end{abstract}

Keywords: EIT, Regularization, Reconstruction Algorithm, Iterative, PD-IPM

\section{Introduction}

A living thing such as an animal or plant is developed with cells and tissues arranged in three dimensional array. For example, human body is a biological subject which is a very complex structure constructed by many living tissues [1-2]. Those living tissues exhibit electrical conductivity or resistivity and conductivity or resistivity change occurs under different circumstances. EIT is a technique of imaging this conductivity change or absolute conductivity. In EIT, current signal is injected to the closed domain under test through different pairs of electrodes called driving electrodes and voltage data are collected from other electrode pairs called sensing electrodes. The voltage data obtained from noninvasive boundary measurements is used to reconstruct the conductivity distribution inside the closed domain [3-10]. Therefore EIT involves two problems: forward problem and inverse problem. The forward problem of EIT involves constructing a model to calculate the potentials (or currents) produced on the boundary, when currents are injected (or voltages are applied) on the same boundary. This model indeed is typically obtained by assuming the geometry and conductivities are known and solving Laplace's equation in the interior of the volume with appropriate boundary conditions [11]. The process of generating image from the measured data is called image reconstruction. Image reconstruction in EIT is referred to as inverse problem. Regarding a forward problem which is simply described as "model parameter" to "data" and an inverse problem can be described as "data" to "model parameter" [13].

EIT has several advantages over other computed tomography imaging techniques used for medical imaging applications. EIT is noninvasive, radiation free, nonionizing method, suitable for the patients of any age as well as critically ill patients, bedside measurement and ICU monitoring, and it is low cost device. Moreover, EIT poses high temporal resolution [12]. 


\section{Reconstruction Algorithm}

Image reconstruction algorithm is the most important parts of EIT system. The boundary potential data collected from the real objects are sent to the PC and are processed to reconstruct the EIT images using the EIT reconstruction algorithm. EIT image reconstruction algorithm generally has two major parts:

- Forward solver

- Inverse solver

The forward solver solves the characteristic equation or governing equation of EIT [12] and computes the boundary potentials called calculated boundary data $V_{c}$ for a known current stimulation. The calculated potential data $V_{c}$ are then compared with the measured potential data $V_{m}$ in inverse solver and the conductivity distribution of the volume to be tested is reconstructed for which the difference between $V_{m}$ and $V_{c}$ is minimized [12]. This is difference Imaging. Absolute imaging is also possible. Due to nonlinearity, EIT requires regularization techniques.

\subsection{TV Regularization}

The TV regularization method has the ability to preserve discontinuities in reconstructed profiles. This property is desirable in many fields of application of EIT imaging, such as the medical and the industrial, where inter-organ boundaries, in the first case, and inter-phase boundaries, in the latter case, present step changes in electrical properties which are difficult to be reconstructed with traditional regularization methods, as they tend to smooth the reconstructed image [13]. The $\mathrm{TV}$ regularization leads to the formulation of the inverse problem as a minimization of a non-differentiable function.

\subsection{PD-IPM Algorithm}

This method is based on the primal-dual theory developed by Andersen [13]. TV regularized inverse problem is formularized as,

$\boldsymbol{\sigma}_{\text {rec }}=\arg \frac{\min }{\boldsymbol{\sigma}} \frac{1}{2}\left(\left\|\mathrm{~F}(\boldsymbol{\sigma})-\mathbf{V}_{\mathrm{m}}\right\|^{2}+\alpha \mathrm{TV}(\boldsymbol{\sigma})\right)$

Where, $F(\boldsymbol{\sigma})$ is the forward model prediction, $\mathbf{V}_{\mathrm{m}}$ is the vector of the measured voltages, $\operatorname{TV}(\boldsymbol{\sigma})$ is the regularization functional, $\alpha$ is a hyperparameter controlling the level of regularization, $\boldsymbol{\sigma}$ is the conductivity vector and the norm $\|\cdot\|$ is the 2-norm [17]. The system of nonlinear equations that defines the PD-IPM method for (1) is written as,

$$
\begin{aligned}
& \left\|y_{i}\right\|<1 \\
& \mathbf{H}^{\mathrm{T}}\left(\mathrm{F}(\boldsymbol{\sigma})-\mathbf{V}_{\mathrm{m}}\right)+\alpha \mathbf{L}^{\mathrm{T}} \boldsymbol{\sigma}=0 \\
& \mathbf{L} \boldsymbol{\sigma}-\mathbf{E} \mathbf{y}=0
\end{aligned}
$$

Where, $\mathbf{y}$ is the primal variable, $\mathbf{L}$ is an approximate regularization matrix, $\mathbf{E}$ is a diagonal matrix and $\mathbf{H}$ is the Jacobian of $\mathrm{F}(\sigma)$. Equation (2) can be solved to obtain the updates $\delta \boldsymbol{\sigma}$ and $\delta \mathbf{y}$ of the primal and dual variables.

$$
\left[\begin{array}{cc}
\mathbf{H}^{\mathrm{T}} \mathbf{H} & \alpha \mathbf{L}^{\mathrm{T}} \\
\mathbf{K L} & -\mathbf{E}
\end{array}\right]\left[\begin{array}{l}
\delta \boldsymbol{\sigma} \\
\delta \mathbf{y}
\end{array}\right]=-\left(\begin{array}{c}
\mathbf{H}^{\mathrm{T}}\left(\mathrm{F}(\boldsymbol{\sigma})-\mathbf{V}_{\mathrm{m}}\right)+\alpha \mathbf{L}^{\mathrm{T}} \mathbf{y} \\
\mathbf{L} \boldsymbol{\sigma}-\mathbf{E y}
\end{array}\right)
$$

Where, $\mathbf{K}=\operatorname{diag}\left(1-\mathbf{y}_{\mathrm{i}} \mathbf{L}_{\mathrm{i}} \boldsymbol{\sigma} / \mathbf{E}(\mathrm{i}, \mathrm{i})\right)$. Equation (3) is solved as follows,

$$
\left[\mathbf{H}^{\mathrm{T}} \mathbf{H}+\alpha \mathbf{L}^{\mathrm{T}} \mathbf{E}^{-1} \mathrm{~h} \mathbf{L}\right] \delta \boldsymbol{\sigma}=-\left[\mathbf{H}^{\mathrm{T}}\left(\mathrm{F}(\boldsymbol{\sigma})-\mathbf{V}_{\mathrm{m}}\right)+\alpha \mathbf{L}^{\mathrm{T}} \mathbf{E}^{-1} \mathbf{L} \boldsymbol{\sigma}\right]
$$


$\delta \mathbf{y}=-\mathbf{y E}^{-1} \mathbf{L} \sigma+\mathbf{E K L} \delta \sigma$

Equations (4) and (5) can therefore be applied iteratively to solve the non-linear inversion of equation (1). The iterative procedure must be initialized which is done by setting $\boldsymbol{y}_{0}=0$. Thus in the first iteration (5) is solved as,

$\delta \boldsymbol{\sigma}=\left(\mathbf{H}^{\mathrm{T}} \mathbf{H}+\boldsymbol{\alpha} \mathbf{L}^{\mathrm{T}} \mathbf{L}\right)^{-1}-\left(\mathbf{H}^{\mathrm{T}}(\mathrm{F}(\boldsymbol{\sigma}))-\mathbf{V}_{\mathrm{m}}\right)$

$\delta \mathbf{y}=-\mathbf{E}^{-1} \mathbf{L} \boldsymbol{\sigma}+\mathbf{E K L} \delta \boldsymbol{\sigma}$

The simplest way to compute the update is called the scaling rule [14],

$\mathbf{y}^{\mathrm{k}+1}=\Phi^{*}\left(\mathbf{y}^{\mathrm{k}}+\delta \mathbf{y}^{\mathrm{k}}\right)$

Where, $\Phi^{*}$ is a scalar value. Now the exact step length by applying the step length rule,

$\mathbf{y}^{\mathrm{k}+1}=\mathbf{y}^{\mathrm{k}}+\min \left(1, \Phi^{*}\right) \delta \mathbf{y}^{\mathrm{k}}$

For larger contrasts a line search on the primal variable guarantees the stability [17].

\section{Simulation Design}

Figure 1 show the geometry used to carry out the simulation. Figure 1(a) is the mesh view and figure 1(b) is the cross-sectional view. This is a Finite Element Method (FEM) model, which contains 576 mesh elements. This FEM model has a radius of $1 \mathrm{~cm}$ and conductivity of $1 \mathrm{~S} / \mathrm{m}$. This is called background. An impurity has a radius of $0.25 \mathrm{~cm}$ and conductivity of $1.5 \mathrm{~S} / \mathrm{m}$ is inserted into this.

The electrodes used here are point electrodes. There are 16 electrodes and this is a complete electrode model. Current applied has a amplitude of $10 \mathrm{~mA}$ and frequency of $1 \mathrm{KHz}$. Adjacent simulation pattern is used with no measurement taken at current stimulation electrodes.

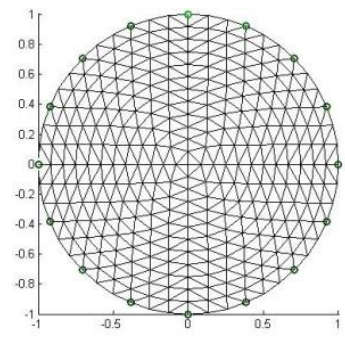

a

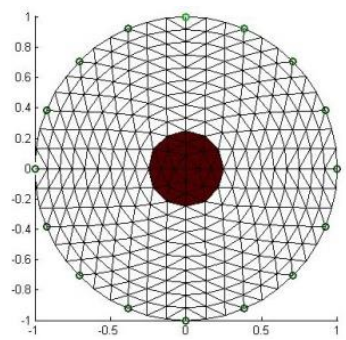

$\mathrm{b}$

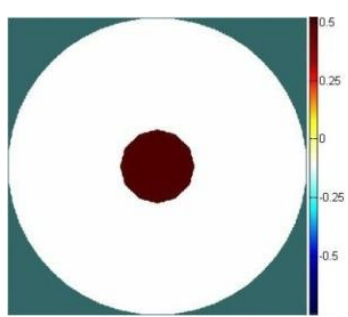

C

Figure 1. Simulation Geometry, (a) Background with Homogeneous Conductivity (b) Impurity Inserted into Background (c) True Difference Conductivity Image

\section{Results}

The resulting difference images obtained with TV regularized PD-IPM algorithm is shown in the following figures. Images are obtained in noise free and noisy cases $(30 \mathrm{~dB}$ and $15 \mathrm{~dB}$ ). This section also contains the calculated percentage of error in conductivity image reconstruction. 


\subsection{Reconstructed Conductivity Images}

Figure 2, 3 and 4 depict the reconstructed conductivity images in noise free case, $30 \mathrm{~dB}$ noisy case and $15 \mathrm{~dB}$ noisy case respectively. Although in each case a total number of 15 iterations are carried out but only four iteration results are inserted.

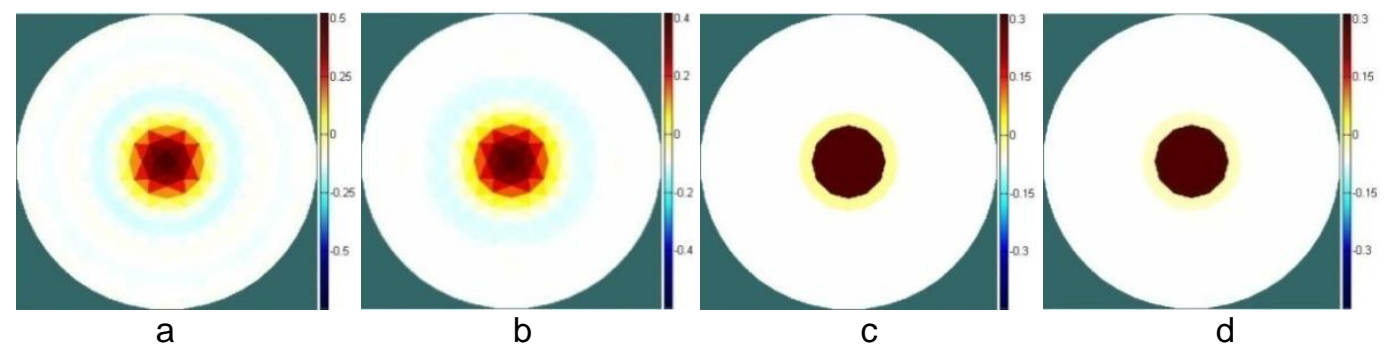

Figure 2. Noise free case, reconstructed conductivity images using TV regularized PD-IPM algorithm, (a) $1^{\text {st }}$ iteration (b) $3^{\text {rd }}$ iteration (c) $10^{\text {th }}$ iteration (d) $15^{\text {th }}$ iteration

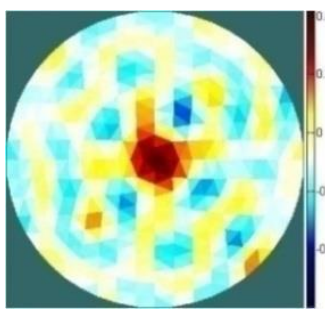

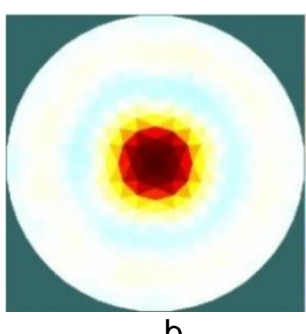

b

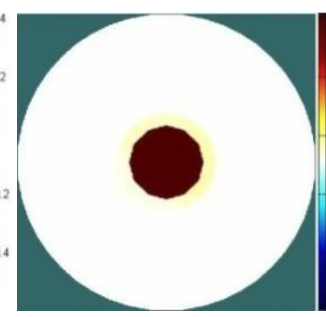

c

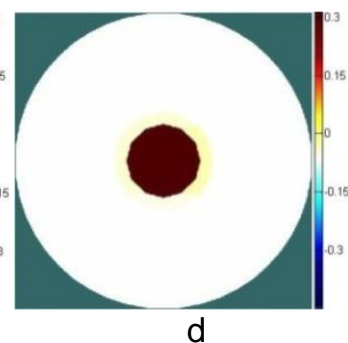

Figure 3. Noisy case (30 dB), reconstructed conductivity images using TV regularized PD-IPM algorithm, (a) $1^{\text {st }}$ iteration (b) $3^{\text {rd }}$ iteration (c) $10^{\text {th }}$ iteration (d) $15^{\text {th }}$ iteration

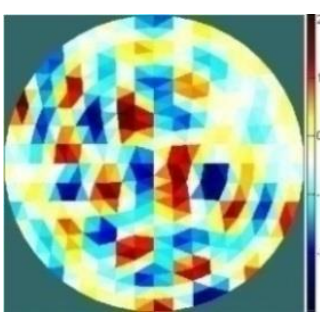

a

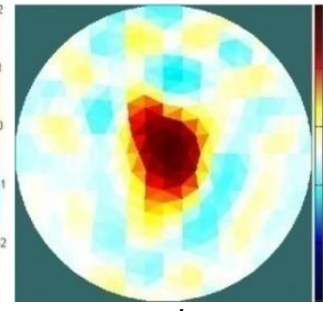

b

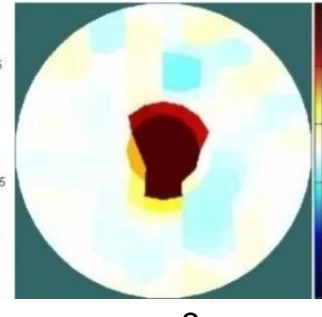

C

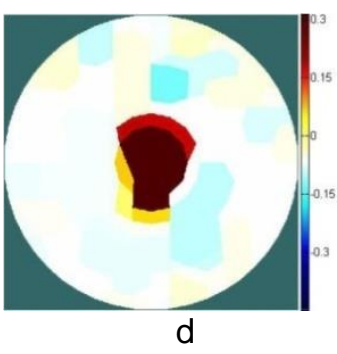

Figure 4. Noisy case (15 dB), reconstructed conductivity images using TV regularized PD-IPM algorithm, (a) $1^{\text {st }}$ iteration (b) $3^{\text {rd }}$ iteration (c) $10^{\text {th }}$ iteration (d) $15^{\text {th }}$ iteration

In noise free case, the results show that TV regularized PD-IPM algorithm has better performance. It is observed that this algorithm converges quickly. The original shape, location and size of the impurity can be recovered with only fifteen iterations. This is much less then number of iteration required in [15]. At $1^{\text {st }}$ and $3^{\text {rd }}$ iteration a conductivity region is generated around the object which is not present in true map. At $15^{\text {th }}$ iteration output image has look same as true map (Figure 1(c)). One most important observation is that, this algorithm is capable to preserve sharp discontinuities between different regions having different conductivities in the reconstructed image and preserve sharp transition in conductivity which agrees with [15]. Edge preservation has clear clinical benefits. The ability thus of reconstructing sharp changes should lead to a better estimation of the boundaries and also to a better accuracy in the estimated values. For example, in the case of lung imaging, sharp boundaries can occur within a diseased lung, at the interface 
between ventilated and collapsed regions. Sharp images therefore allow to better identification of the boundary between the two regions. Again, there is almost no blurring effect. This is because TV regularization is used. In TV regularization to reduce the blurring effect, an "image enhancement" technique was applied to the EIT problem [16].

This algorithm is very sensitive to noise. Faithful reconstruction of image is possible only with SNR equal to or above $30 \mathrm{~dB}$. With this condition the algorithm is capable to recover exact shape, size, spatial location and sharp discontinuities at higher iteration number as shown in figure 3. Algorithm performance decreases with decreasing SNR. One important observation is that, even when the error grows as the SNR decreases, the spatial location of the object remains quite acceptable.

Although reconstructed image quality is better, one disadvantage is that, since it is iterative, it needs a longer computation time and huge resources in order to reconstruct conductivity image. Furthermore, this algorithm is more complex to implement and carry out large number of iterations huge storage capacity is needed.

\subsection{Error in Conductivity Image Reconstruction}

The calculated percentage of error in the reconstruction of conductivity change with respect to the true conductivity is given in the table 1 .

Table 10. Error in Conductivity Image Reconstruction

\begin{tabular}{|c|c|c|c|}
\hline \multirow[b]{2}{*}{ Case } & \multirow[b]{2}{*}{ Iteration no. } & \multicolumn{2}{|c|}{ Error } \\
\hline & & $\begin{array}{c}\text { Impurity } \\
(\%)\end{array}$ & $\begin{array}{c}\text { Background } \\
(\%)\end{array}$ \\
\hline \multirow{4}{*}{ Noise free } & $1^{\mathrm{st}}$ & 10.87 & 1.11 \\
\hline & $3^{\text {rd }}$ & 12.00 & 1.07 \\
\hline & $10^{\text {th }}$ & 8.91 & 0.30 \\
\hline & $15^{\text {th }}$ & 8.38 & 0.19 \\
\hline \multirow{4}{*}{$\begin{array}{c}\text { Noisy } \\
\text { (30 dB) }\end{array}$} & $1^{\mathrm{st}}$ & 12.10 & 8.44 \\
\hline & $3^{\text {rd }}$ & 12.97 & 1.57 \\
\hline & $10^{\text {th }}$ & 8.08 & 0.18 \\
\hline & $15^{\text {th }}$ & 7.87 & 0.14 \\
\hline \multirow{4}{*}{$\begin{array}{c}\text { Noisy } \\
(15 \mathrm{~dB})\end{array}$} & $1^{\mathrm{st}}$ & 71.36 & 65.64 \\
\hline & $3^{\text {rd }}$ & 14.48 & 3.37 \\
\hline & $10^{\text {th }}$ & 13.40 & 1.61 \\
\hline & $15^{\text {th }}$ & 13.38 & 1.58 \\
\hline
\end{tabular}

\section{Conclusion}

EIT is a most promising biomedical imaging technique. Large difference between different biological tissues makes this technique very much attractive but this technique is still under laboratory research. In this study a reconstruction algorithm for EIT have been studied to test their practical applicability in the noise free case and noisy case. The TV regularized PD-IPM algorithm provides the relatively low error in image reconstruction both in noise free and noisy case. Moreover, all other criteria such as recovery of size, shape and spatial location were found best for TV regularized PD-IPM algorithm. But in PD-IPM algorithm large number of iteration is needed. Here, only one impurity has been considered, two or more impurities can be considered in a similar way. The number of mesh elements in the forward model affects the size and shape of the impurity. Moreover, the conductivity distribution depends on the material of electrodes, number of electrodes and the medium used in the experiment can be studied in future. 


\section{Acknowledgment}

The authors thank the members of the Department of Biomedical Physics and Technology, DU.

\section{References}

[1] T. K. Bera and N. Jampana, "A Multifrequency Constant Current Source for Medical Electrical Impedance Tomography”, IEEE International Conference on Systems in Medicine and Biology, Kharagpur, India, (2010), pp. 290-295.

[2] F. H. Netter, "Atlas of Human Anatomy", Rittenhouse, Book Distributors Inc, 2nd edition, (1997).

[3] T. I. Oh, H. Koo and K. H. Lee, "Validation of a Multifrequency Electrical Impedance Tomography (mfEIT) System KHU Mark1: Impedance Spectroscopy and Time-Difference Imaging”, Physiological Measurement, vol. 29, no. 3, (2008), pp. 295-307.

[4] T. K. Bera and J. Nagaraju, "A Study of Practical Biological Phantoms with Simple Instrumentation for Electrical Impedance Tomography (EIT)", IEEE Instrumentation and Measurement Technology Conference, Singapore, (2009), pp. 511- 516.

[5] P. J. Riu, J. Rosell, A. Lozano, and R. Pallas-Areny, "Multifrequency Static Imaging in Electrical Impedance Tomography: Part 1 Instrumentation Requirements", Medical and Biological Engineering and Computing, vol. 33, no. 6, (1995), pp. 784-792.

[6] T. K. Bera, M. Saikia, and J. Nagaraju, "A Battery-Based Constant Current Source (Bb-CCS) for Biomedical Applications", International Conference on Computing, Communication and Networking Technologies Tamilnadu, India, (2013).

[7] T. I. Oh, H. Wi, P. J. Yoo, and E. J. Woo, "A Fully Parallel Multi-frequency EIT system with Flexible Electrode Configuration: KHU Mark2”, Physiological Measurement, vol. 32, no. 7, (2011), pp. 835-849.

[8] T. K. Bera and J. Nagaraju, "A Lab VIEW Based Multifunction Multifrequency Electrical Impedance Tomography (MfMf-EIT) Instrumentation for Flexible and Versatile Impedance Imaging", 15th International Conference on Electrical Bio-Impedance, Berlin, Germany, (2013), pp. 216.

[9] K. G. Boone and D. S. Holder, "Current Approaches to Analogue Instrumentation Design in Electrical Impedance Tomography”, Physiological Measurement, vol. 17, no. 4, (1996), pp. 229-247.

[10] T. K. Bera and J. Nagaraju, "A Battery Based Multifrequency Electrical Impedance Tomography (BbMfEIT) System for Impedance Imaging of Human Anatomy," 15th International Conference on Electrical Bio-Impedance (ICEBI), Berlin, Germany, (2013), pp. 217.

[11] J. C. Newell, G. D. Gisser and D. Isaacson, "An Electric Current Tomography", IEEE Transactions on Biomedical Engineering, vol. 35, No. 10, (1988).

[12] T. k. Bera, "Bioelectrical Impedance Methods for Noninvasive Health Monitoring: A Review", Hindawi Publishing Corporation Journal of Medical Engineering Volume, Article ID 381251, (2014).

[13] K. D. Andersen, E. Christiansen, A. Conn, and M. L. Overton, "An Efficient Primal-Dual Interior-Point Method for Minimizing a Sum of Euclidean Norms", SIAM J. on Scientific Computing, vol. 22.

[14] N. J. Avis, "Image Reconstruction in Electrical Impedance Tomography", Ph.D. d. dissertation, University of Sheffield, (1993).

[15] A. Borsic, B. M. Graham, A. Adler and W. R. B. Lionhear, "In vivo Impedance Imaging with Total Variation Regularization".

[16] D. C. Dobson, "Recovery of Blocky Images in Electrical Impedance Tomography".

[17] A. Borsic, B. M. Graham, A. Adler and W. R. B. Lionheart, "Total Variation Regularization in Electrical Impedance Tomography", (2007).

\section{Author}

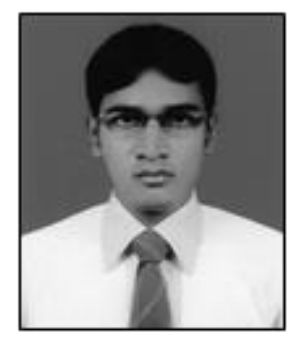

Md. Rabiul Islam was born on $2^{\text {nd }}$ October 1988. He received his B. Sc. (Hon's) degree and M. Sc. degree from Dhaka University in 2010 and 2011 respectively. His main research area is Biomedical Physics. 